Copyright, 195., by the American Rocket Society, Inc., and reprinted by permission of the copyright owner.

\section{Thermodynamic Properties of Gas at High Temperatures and Pressures}

\section{H. S. TSIEN ${ }^{1}$}

Daniel and Florence Guggenheim Jet Propulsion Center, California Institute of Technology, Pasadena, Calif.

\section{Equation of States of Dense Gas}

W HEN the density of gas is high, it is well known that the simple equation of states for a perfect gas can no longer be expected to be valid. The most crude approximation to the equation of states for a dense gas is that of Van der Waal. If $P$ is the pressure, $v$ the volume per molecule, $T$ the temperature, and $k$ the Boltzmann constant, then the Van der Waal equation is

$$
\left(P+\frac{a}{v^{2}}\right)(v-b)=k T
$$

where $a$ and $b$ are two constants, small in magnitude. The constant $b$ is usually simply identified as four times the volume of a molecule. If the molecules are assumed to be spheres of diameter $D$, then

$$
b=\frac{2 \pi}{3} D^{3}
$$

At high temperatures, the density of gas can be large only if the pressure is very high. Then the term $a / v^{2}$ is not important in comparison with $P$, and Equation [1 ] can be simplified into the so-called covolume equation of states

$$
P(v-b)=k T
$$

Or we can write

$$
\frac{P v}{k T}=1+\frac{1}{\frac{3}{2 \pi} \frac{v}{v^{*}}-1} \ldots \ldots \ldots \ldots \ldots[3]
$$

where $v^{*}$ is a volume defined by

$$
v^{*}=D^{3} \text {. }
$$

However, because of the crude approximation in the Van der Waal equation of states, neither Equation [1] nor Equation [2] can be expected to be sufficiently accurate for gas at very high temperatures and high pressures. An example of such state of matter is the gaseous products of detonation of condensed explosives, where temperatures of several thousand degrees Kelvin and densities of the order of solids occur. Other more elaborate equations of states designed to cover the whole range of pressures and temperatures suffer from the same defect.

For products of detonation of condensed explosives, a more accurate equation is the Halford-Kistiakowsky-Wilson equation $(1)^{2}$

$$
\frac{P v}{k T}=1+K T^{-1 / 4} \exp \left(0.3 K / T^{1 / 4}\right)
$$

where

$$
K=\sum_{i} n_{i} K_{i} \ldots \ldots
$$

Received June 20, 1954

1 Director, Guggenheim Jet Propulsion Center.

${ }^{2}$ Numbers in parentheses indicate References at end of paper. $n_{i}$ is the number of moles of the $i$ th molecular species per unit volume, and $K_{i}$ is the empirical constant for the $i$ th species. For water and ammonia, the $K_{i}$ 's are 108 and $164 \mathrm{~cm}^{3}$ per mole, respectively. It is suggested that when $K_{i}$ is not known, it can be calculated as

$$
K_{i}=5.5\left(\frac{2 \pi}{3}\right) N D_{i}{ }^{3} \ldots \ldots \ldots \ldots \ldots
$$

where $N$ is Avogadro's number, and $D_{i}$ is the low energy collision diameter of molecules of the $i$ th species. $K_{i}$ is thus equal to 22 times the volume of one mole of spherical molecules of diameter $D_{i}$. If there is only one species of molecules, then Equations [6] and [7] give

$$
K=5.5\left(\frac{2 \pi}{3}\right) /\left(\frac{v}{v^{*}}\right)
$$

where $v^{*}$ is a volume defined by Equation [4]. Thus for a single gas, the Halford-Kistiakowsky-Wilson equation can be written as

$$
\frac{P v}{k T}=1+\frac{11.51}{\left(\frac{v}{v^{*}}\right) T^{1 / 4}} \exp \left(\frac{3.453}{\frac{v}{v^{*}} T^{1 / 4}}\right)
$$

It is easily seen from Equations [5] and [9] that they are an improvement over the covolume equation [3]; now the "compressibility," $P b / k T$, is unity as $T \rightarrow \infty$ even with $v / v^{*}$ finite. This result is to be expected on the general ground that molecules are never rigid spheres, but "squeezy," i.e., closer approach is possible if two molecules collide with greater kinetic energy. At very high temperatures, the kinetic energy of the molecules is very high, then the effective size of the molecules in collision must be very small. Now the effects of gas imperfection is proportional to the molecular size. Therefore as $T \rightarrow \infty$, the effective molecular size vanishes and the gas becomes a perfect gas, even if the volume ratio is finite. Hence really satisfactory equation of states must have this property. This is so for the Halford-Kistiakowsky-Wilson equation.

But even when the constant $K$ is related to the low energy collision diameter of the molecules through Equations [7] and [8], still no proper account is made for the strength of molecular interaction. If we represent the interaction between a pair of molecules by the Lennard-Jones potential $\epsilon(r)$, a function of the intermolecular distance $r$

$$
\epsilon(r)=4 \epsilon^{*}\left[\left(\frac{D}{r}\right)^{12}-\left(\frac{D}{r}\right)^{6}\right]
$$

then besides the collision diameter $D$, there is the parameter of equilibrium potential $-\epsilon^{*}$, the potential when the pair of molecules are at their equilibrium distance $2^{1 / 6} D$. A convenient parameter for $\epsilon^{*}$ is the "characteristic temperature of interaction," $\theta_{1}$, defined as

$$
\theta_{1}=\frac{\epsilon^{*}}{k}
$$

Then we expect the equation of states to be of the form

$$
\frac{P v}{k T}=1+f\left(T / \Theta_{1}, v / v^{*}\right) \ldots
$$

i.e., the compressibility should be a function of temperature ratio $T / \theta_{1}$, but not temperature itself as is in the HalfordKistiakowsky-Wilson equation. Furthermore, according to our argument on vanishing imperfection at $T \rightarrow \infty$

$$
f\left(\infty, v / v^{*}\right)=0 .
$$


With the'general concept that the equation of states for dense gas must satisfy the conditions embodied in Equations [12] and [13], many of the equations of states proposed by various authors can be ruled out as unreliable. For instance, Cottrell and Paterson suggested (2) that

$$
\frac{P v}{k T}=3+\frac{\text { const }}{\left(T / \theta_{1}\right)^{1 / 2}\left(\frac{v}{v^{*}}\right)}
$$

Since the condition of Equation [13] is not satisfied, this equation cannot be reliable for very high temperatures. In a similar manner, the theoretical equation of states proposed by Zwansig (3) is also unacceptable, because he makes the untenable assumption that at very high temperatures the molecules interact as rigid spheres.

\section{Lennard-Jones and Devonshire Theory}

If there were sufficient experimental data for gas at very high temperatures and pressures, we can try to fit the data to the nondimensional equation of states of Equation [12] and determine the function $f\left(T / \Theta_{1}, v / v^{*}\right)$. This is, in effect, the application of the principle of corresponding states. Unfortunately, except for two old measurements on hydrogen and helium by Bridgman (4), no experimental data are available at high enough temperatures and pressures for this to be possible. We must then turn to theory to determine the proper equation of states. If we are interested in the region of density where the nondimensional volume $v / v^{*}$ is near unity, i.e., densities of the order of liquid density at low pressure, then the Lennard-Jones and Devonshire theory of liquids and dense gas is a very good approximation to the true physical situation. Here the main defect of not allowing for empty lattice sites in the theory is not important. Furthermore, Wentorf, Buhler, Hirschfelder, and Curtiss (5) have carried out very extensive and accurate calculations for the LennardJones and Devonshire theory. We can use their tabulated values directly. But for practical calculations, it would be more convenient to put their result in a simple analytic form for easy interpolation and extrapolation. This is the main purpose of the present note.

Of course theoretically the equation of states can be directly obtained from the formulation of Lennard-Jones and Devonshire theory without having to use the numerical results. But the interested range of parameters $T / \theta_{1}$ and $v / v^{*}$ is such that no simple analytic result can be obtained. This is quite different from the case of liquid state where $T / \theta_{1}$ is small enough for the simple analytic treatment (6) to be successful.

By using the tabulated values of $P v / k T$ given by Wentorf and collaborators, we can plot $T / \Theta_{1}$ against $v / v^{*}$ for constant $P v / k T$. The result of this preliminary investigation indicates that instead of two variables $T / \theta_{1}$ and $v / v^{*}$ in $f$ of Equation [12], a single variable $\eta$ is sufficient with

$$
\eta=\left(T / \Theta_{1}\right)^{1 / 6}\left(v / v^{*}\right)
$$

Then, according to Equations [12] and [13]

$$
\frac{P v}{k T}=1+f(\eta), \quad f(\infty)=0
$$

In Fig. $1,1 / f=(P v / k T-1)^{-1}$ is plotted against $\eta$. We see that the points are grouped around a straight line for $T / \Theta_{1}=$ $10,20,50,100$, and 400 and $v / v^{*}$ near unity. In fact, we find the approximate equation of states for gas at very high temperatures and pressures as

$$
\frac{P v}{k T}=1+\frac{1}{0.278 \eta-0.177}
$$

The condition of Equation [13] is thus satisfied. This result is accurate to about 10 per cent.

Although the equation of states, Equation [17], is obtained for dense gas at high temperature, it may be interesting to see how well it behaves for dilute gas. For dilute gas, $v / v^{*}$ is

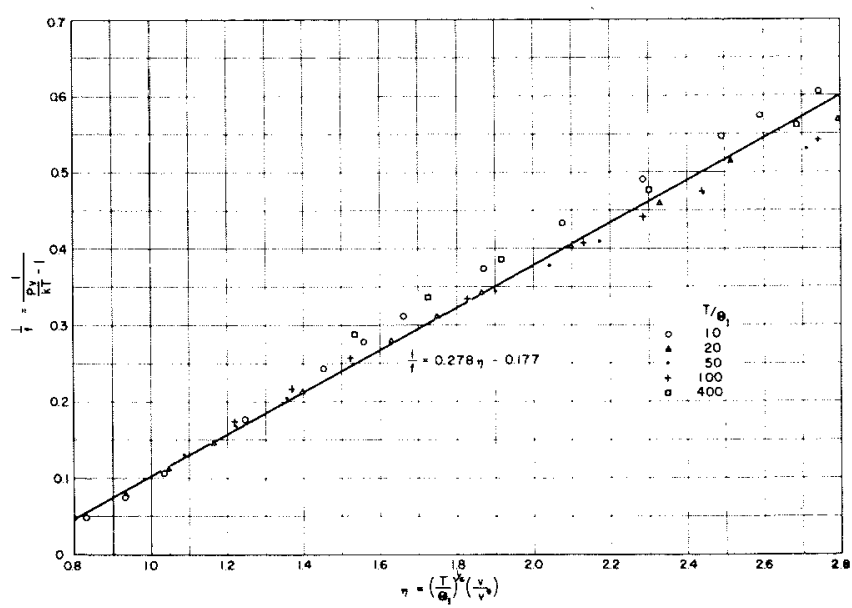

Fig. 1

very large, then $\eta$ is very large. Therefore for dilute gas at high temperatures, we have

$$
\frac{P v}{k T} \cong 1+\frac{1}{0.278 \eta}=1+3.594\left(T / \theta_{1}\right)^{-1 / 6}\left(v / v^{*}\right)^{-1}
$$

On the other hand, the exact equation of states for dilute gas at very high temperatures is given by the virial equation, retaining only the leading term in the expansion of the second virial coefficient (7). Thus

$$
\begin{aligned}
\frac{P v}{k T} \cong 1+\frac{2 \sqrt{2} \pi}{3} \Gamma\left(\frac{3}{4}\right)\left(T / \Theta_{1}\right)^{-1 / 4}\left(v / v^{*}\right)^{-1}= \\
1+3.630\left(T / \theta_{1}\right)^{-1 / 4}\left(v / v^{*}\right)^{-1}
\end{aligned}
$$

By comparing Equations [18] and [19], it is seen that aside from the difference in the exponent of $T / \theta_{1}$, our approximate equation of dense gas at high temperatures even reproduces some of the characteristics of a dilute gas. Therefore for rough approximations, our equation of states, Equation [17], can be even used for the complete range of $v / v^{*}$. Of course, Equation [17] can be true only for high temperatures, say $T / \theta_{\mathrm{t}}>10$.

\section{Other Thermodynamic Functions}

Since the proposed equation of states is approximately true for even dilute gas, we may use it to calculate other thermodynamic functions by integrating to $v / v^{*} \rightarrow \infty$. For instance, if $E$ is the energy per molecule, then the general thermodynamic law states that

$$
\left(\frac{\partial E}{\partial v}\right)_{T}=T\left(\frac{\partial P}{\partial T}\right)_{v}-P
$$

Thus, by differentiating Equation [17], using Equation [15]

$$
\left(\frac{\partial E}{\partial v}\right)_{T}=-\frac{k T}{v} \frac{0.278 \eta}{(0.278 \eta-0.177)^{2}} \ldots \ldots \ldots
$$

Thus, if $E_{\infty}$ is the energy per molecule when $v / v^{*} \rightarrow \infty$, i.e., $E_{\infty}$ is the energy per molecule calculated for a perfect gas, then the energy per molecule due to imperfection of the gas is

$$
\left(E-E_{\infty}\right)_{T}=-\int_{v}^{\infty}\left(\frac{\partial E}{\partial v}\right)_{T} d v \ldots \ldots \ldots[21]
$$

By substituting Equation [20] into Equation [21], and by considering $\left(E-E_{\infty}\right) / k T$ to be a function of $\eta$ only, we obtain

$$
\left(E-E_{\infty}\right)_{T}=\frac{k T}{6} \frac{1}{0.278 \eta-0.177}
$$

We observe that although the result involves the use of our approximate equation of states for large $v / v^{*}$, the contribu-

(Continued on page 478) 


\section{Thermodynamic Properties of Gas}

(Continued from page 472)

tion to $E-E_{\infty}$ at large $v / v^{*}$ is small, and the error made is not as serious as one might conclude at first sight. In fact, by comparing the computed value using Equation [22] with the exact numerical value of the same function tabulated by Wentorf and collaborators, the difference is of the order of 10 per cent. Ten per cent accuracy is all we claim for the equation of state.

Similarly, we have the following formulas for molecular enthalpy $H$, molecular heat capacities, $c_{v}$ and $c_{\mathcal{D}}$, molecular entropy $s$

$$
\begin{gathered}
\left(H-H_{\infty}\right)_{T}=7\left(E-E_{\infty}\right)_{T} \ldots \ldots \ldots[23] \\
\left(c_{v}-c_{v_{\infty}}\right)_{T}=\frac{k}{6} \frac{0.2314 \eta-0.177}{(0.278 \eta-0.177)^{2}} \ldots \ldots \ldots[24] \\
\left(c_{p}-c_{p_{\infty}}\right)_{T}=7\left(c_{1}-c_{v_{\infty}}\right)_{T} \ldots \ldots \ldots[25]
\end{gathered}
$$

$\left(s-s_{\infty}\right)_{T}=\frac{k}{6} \frac{1}{0.278 \eta-0.177}-$

$$
\frac{k}{0.177} \log \frac{0.278 \eta}{0.278 \eta-0.177} \ldots
$$

The above formulas for heat capacities and entropy are less accurate than for energy and enthalpy because of the differentiation used in their derivations. But these equations are at least consistent among themselves.

\section{References}

1 "Molecular Theory of Gases and Liquids," by J. O. Hirschfelder, C. F. Curtiss, and R. B. Bird, John Wiley, 1954, p. 263.

2 "An Equation of State Applicable to Gases at Densities Near That of the Solid and Temperatures far Above the Critical," by T. L. Cottrell and S. S. Paterson, Proc. Roy. Soc. $(A)$ vol. 213, p. 214, 1952.

3 "High-Temperatures Equation of State by a Perturbation Method. I. Nonpolar Gases," by R. W. Zwansig, J. Chem. Phys., vol. 22, p. 1420, 1954 .

4 "The Physics of High Pressures," by P. W. Bridgman, G. Bell and Sons, 1931, p. 108.

5 "Lennard-Jones and Devonshire Equation of State of Compressed Gases and Liquids," by R. H. Wentorf, R. J. Buhler, J. O. Hirschfelder, and C. F. Curtiss, J. Chem. Phys., vol. 18, p. 1484, 1950.

6 "The Properties of Pure Liquids," by H. S. Tsien, Journal of the American Rocket Society, January-February 1953.

7 Ref. 1, p. 163. 